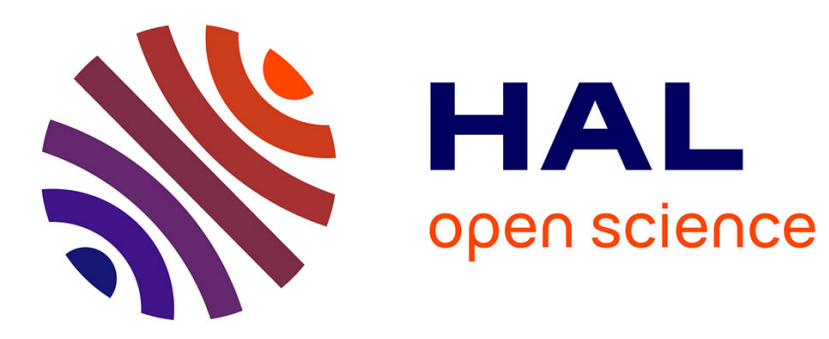

\title{
Efficacité et robustesse aux distracteurs d'un retour tactile pour faciliter le pointage
}

Géry Casiez, Nicolas Roussel, Romuald Vanbelleghem, Frédéric Giraud

\section{To cite this version:}

Géry Casiez, Nicolas Roussel, Romuald Vanbelleghem, Frédéric Giraud. Efficacité et robustesse aux distracteurs d'un retour tactile pour faciliter le pointage. IHM '10: Conference Internationale Francophone sur I'Interaction Homme-Machine, AFIHM, Sep 2010, Luxembourg, Luxembourg. pp.25-32, 10.1145/1941007.1941012 . inria-00533998

\section{HAL Id: inria-00533998 \\ https://inria.hal.science/inria-00533998}

Submitted on 15 Nov 2010

HAL is a multi-disciplinary open access archive for the deposit and dissemination of scientific research documents, whether they are published or not. The documents may come from teaching and research institutions in France or abroad, or from public or private research centers.
L'archive ouverte pluridisciplinaire HAL, est destinée au dépôt et à la diffusion de documents scientifiques de niveau recherche, publiés ou non, émanant des établissements d'enseignement et de recherche français ou étrangers, des laboratoires publics ou privés. 


\title{
Efficacité et robustesse aux distracteurs d'un retour tactile pour faciliter le pointage
}

\author{
Géry Casiez ${ }^{1,3,4}$, Nicolas Roussel ${ }^{3}$, Romuald Vanbelleghem ${ }^{3}$ \& Frédéric Giraud Rri, $^{2,3}$ \\ ${ }^{1}$ LIFL, ${ }^{2}$ L2EP, ${ }^{3}$ INRIA Lille \& ${ }^{4}$ Université de Lille 1, FRANCE \\ gery.casiez@lifl.fr, nicolas.roussel@inria.fr \\ romuald.vanbelleghem@inria.fr, frederic.giraud@polytech-lille.fr
}

\begin{abstract}
RÉSUMÉ
Surfpad est une technique de facilitation du pointage tirant parti de la possibilité de modifier le coefficient de friction d'un pavé tactile. Nous décrivons deux expériences comparant cette technique au pointage sémantique et à un gain control-display constant, avec et sans distracteurs. Nos résultats montrent les limites de l'adaptation de gain sensible aux cibles en présence de distracteurs et les avantages de notre approche tactile dans les deux cas. Le gain de temps permis par Surfpad face au pointage sans assistance peut aller jusqu'à $21 \%$ dans le cas de petites cibles sans distracteur. Notre technique est également robuste aux grandes densités de distracteurs, améliorant en moyenne les performances de près de $10 \%$ quand le pointage sémantique peut les dégrader jusqu'à $100 \%$.
\end{abstract}

MOTS CLÉS : Facilitation du pointage, pavé tactile, effet de film comprimé, friction, distracteur

\begin{abstract}
Surfpad is a pointing facilitation technique that operates in the tactile domain by taking advantage of the ability to alter a touchpad's coefficient of friction. We report on two experiments comparing it to the semantic pointing technique and constant control-display gain with and without distractor targets. Our results clearly show the limits of traditional target-aware gain adaptation in the latter case, and the benefits of our tactile approach in both cases. Surfpad can lead to a performance improvement of up to $21 \%$ compared to unassisted pointing at small targets with no distractor. It is also robust to high distractor densities, keeping an average performance improvement of nearly $10 \%$ while semantic pointing can degrade up to $100 \%$.
\end{abstract}

CATEGORIES AND SUBJECT DESCRIPTORS: H.5.2 [Information interfaces and presentation] : User interfaces

GENERAL TERMS: Human Factors, Performance

Permission to make digital or hard copies of all or part of this work for personal or classroom use is granted without fee provided that copies are not made or distributed for profit or commercial advantage and that copies bear this notice and the full citation on the first page. To copy otherwise, to republish, to post on servers or to redistribute to lists, requires prior specific permission and/or a fee.

IHM 2010, 20-23 September 2010, Luxembourg, Luxembourg
KEYWORDS: Pointing facilitation, touchpad, squeeze film effect, friction, distractor

\section{INTRODUCTION}

Le pointage est une des tâches fondamentales de l'interaction homme-machine et a fait l'objet de nombreuses études. La loi de Fitts est l'un des modèles les plus robustes et les plus utilisés dans ce contexte [3]. Cette loi exprime le temps requis pour atteindre une cible de largeur $W$ à une distance $D$ comme une fonction linéaire de l'indice de difficulté $I D=\log _{2}\left(\frac{D}{W}+1\right)$.

De nombreuses techniques ont été proposées visant à battre la loi de Fitts dans les mondes virtuels [3]. Elles tentent généralement de réduire $D$, d'augmenter $W$, ou combinent ces deux approches. La plupart sont sensibles aux cibles : elles utilisent des connaissances sur leur position et leur taille et les modifient parfois. Dans les cas de pointage indirect, certaines jouent sur le gain controldisplay liant les déplacements du dispositif et du pointeur (gain $=V_{\text {pointeur }} / V_{\text {dispositif }}$ ). D'autres opèrent dans le domaine sonore ou haptique. Mais bien que l'efficacité théorique de ces techniques ait été démontrée, leur mise en pratique reste difficile, posant notamment la question des interférences créées par les autres cibles sur le chemin de celle visée (appelées distracteurs dans ce qui suit).

Dans cet article, nous présentons Surfpad, une technique de facilitation du pointage qui ne modifie ni $D$ ni $W$, ni dans l'espace moteur ni dans l'espace visuel. Surfpad opère dans le domaine haptique en modifiant le coefficient de friction d'un pavé tactile, le STIMTAC [5], à l'aide d'un effet de film comprimé. Nous décrivons deux expériences comparant Surfpad au pointage sémantique [6] et à un gain constant, avec et sans distracteurs. Nos résultats montrent les limites de l'adaptation de gain sensible aux cibles en présence de distracteurs et les avantages de notre approche tactile dans les deux cas. Nous concluons par une discussion de ces résultats et quelques pistes pour des travaux futurs.

\section{TRAVAUX ANTÉRIEURS}

Balakrishnan [3] proposant une revue détaillée de nombreuses techniques de pointage, nous ne retenons dans la sous-section suivante que quelques exemples significatifs. 


\section{Réduire $D$ et augmenter $W$}

Diverses méthodes ont été proposées pour réduire la distance à la cible. Drag-and-pop [4] rapproche temporairement les cibles potentielles du pointeur, par exemple. D'autres techniques utilisent la prédiction de cible pour faire sauter le pointeur au-dessus des espaces vides et des distracteurs [14]. Diverses méthodes ont également été proposées pour augmenter la taille des cibles. Les cibles grossissantes offrent ainsi un espace d'interaction plus grand à l'approche du pointeur, ce grossissement intervenant dans l'espace visuel. Des travaux ont également montré que la loi de Fitts reste valide si l'on considère la taille du pointeur et non celle de la cible, donnant lieu à la création de pointeurs à large zone d'activation [22,8].

Un problème commun aux techniques évoquées est qu'elles peuvent être visuellement perturbantes en raison des déplacements ou redimensionnements qu'elles occasionnent. Une solution à ce problème consiste à intervenir dans l'espace moteur plutôt que visuel. Décrites sous le nom de pointage sémantique [6] ou en termes de champs de force ou de matériaux collants, différentes techniques opèrent ainsi en modifiant le gain $[22,10,6]$ ou en déplaçant artificiellement le pointeur [10,9]. Le gain est typiquement réduit à l'approche des cibles, les agrandissant dans l'espace moteur, tandis que le déplacement du curseur permet des ajustements de trajectoire arbitraires.

Keyson [17] propose une utilisation originale de l'adaptation de gain pour créer un effet d'accroche. En imposant un mouvement plus grand pour sortir de la cible que pour y entrer sans changer le mouvement nécessaire pour la traverser, sa technique préserve l'indice de difficulté des tâches de pointage. L'adaptation de gain peut être vue ici comme un moyen de retourner de l'information plutôt que d'optimiser les paramètres de la loi de Fitts, une approche à la base du retour pseudo-haptique [18].

\section{Utilisation du retour haptique pour faciliter le pointage}

La modalité haptique est depuis longtemps utilisée pour suppléer ou compléter la modalité visuelle dans des situations particulières liées au handicap ou à la téléopération, par exemple. Divers travaux ont également porté sur son utilisation dans un contexte plus général pour faciliter l'acquisition de cibles dans les interfaces graphiques.

Les tâches de pointage discret $2 D$ sont celles qui ont reçu le plus d'attention $[2,1,17,19,12,16]$. Le pointage réciproque $1 D$ [9], le pointage réciproque $2 D$ et le franchissement [13], la traversée de tunnel [7,11] ainsi que des tâches de nature écologique $[19,11,9]$ ont également été étudiés. Le dispositif le plus utilisé pour ce type d'étude est une souris, les autres dispositifs incluant un trackpoint [7], un stylo [13], un trackball [17], un joystick [16] et un dispositif à six degrés de liberté [19]. Le retour haptique est fourni en exerçant une force sur le dispositif pour contraindre son mouvement $[1,17,19,11,12,16]$ ou en déplaçant ou faisant vibrer un ou plusieurs de ses éléments $[2,1,7,9,13]$.
Le pointage avec retour haptique a été principalement comparé au pointage sans retour d'information. Toutes les études ont montré un effet positif, le retour haptique réduisant le temps de pointage $[1,7,11,12,16,9,13]$, le temps pour s'arrêter sur la cible [2] ou le taux d'erreur [19]. Le déplacement ou la vibration de parties mécaniques pouvant générer des sons audibles, des précautions ont souvent été prises pour les masquer, le retour auditif et le retour haptique produisant des effets similairement positifs $[2,9]$. Le retour haptique a également été comparé au retour visuel [2, 7] et à l'adaptation de gain [9], et des chercheurs se sont intéressés à la combinaisons des différentes modalités [2, 1, 7, 9].

Les dispositifs haptiques cités sont tous basés sur des technologies électromagnétiques simples et éprouvées (e.g. solénoïdes, moteurs vibrants et masselottes). La gamme de sensations haptiques permises est en contrepartie très limitée. De nouvelles technologies haptiques offrent toutefois des perspectives intéressantes pour l'interaction homme-machine en général, et la facilitation du pointage en particulier. Ainsi, alors que la plupart des retours tactiles existants reposent sur une stimulation active de la peau par des éléments mécaniques, Watanabe \& Fukui ont proposé une méthode permettant d'adoucir le contact avec une surface en la faisant vibrer avec une très faible amplitude mais à très haute fréquence [20]. Cette sensation de contact plus doux est causée par un effet de film comprimé (Figure 1), un effet récemment utilisé avec succès pour simuler des trous et bosses sur une surface [21].
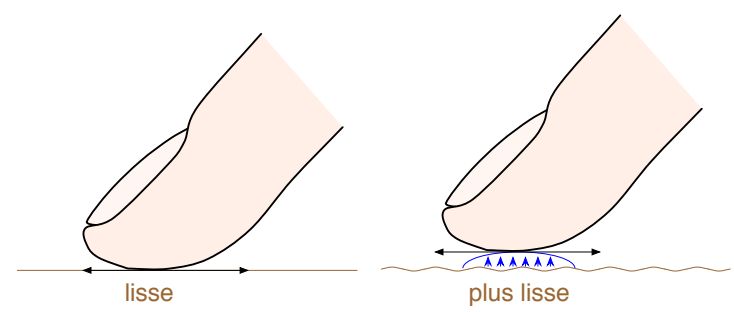

FIGURE 1 : La vibration contrôlée d'une surface crée un film d'air qui réduit son coefficient de friction.

Contrairement aux dispositifs vibrotactiles classiques, ceux basés sur l'effet de film comprimé fournissent un retour passif : ils ne transfèrent pas d'énergie mais modifient la manière dont elle se dissipe au niveau de la zone de contact lors d'un processus de friction initié par l'utilisateur, agissant comme des afficheurs de textures [15].

\section{Impact des distracteurs}

Les techniques de pointage sensibles aux cibles sont $a$ priori plus efficaces lorsque celles-ci sont espacées [3]. Dans la réalité cependant, il n'est pas rare d'observer des groupes de cibles relativement denses. Bien que ce problème soit clairement identifié dans la littérature, peu de recherches ont été menées pour évaluer l'impact exact des distracteurs sur les techniques existantes, ou pour en concevoir de nouvelles les prenant spécifiquement en compte. Parmi les études citées sur le retour haptique et les techniques agissant dans l'espace moteur, quelques unes 
seulement prennent en compte les distracteurs, et de manière très limitée.

Certaines conditions des expériences décrites dans [17] et [22] impliquaient un distracteur incontournable sur le chemin de la cible. Une condition de l'expérience de [16] impliquait un distracteur placé à $0,90,180$ ou $270^{\circ}$ autour de la cible par rapport à la direction du pointage. Dans [12], l'utilisateur devait pointer alternativement une cible parmi 13 disposées en croix, les autres jouant le rôle de distracteurs contournables. Cockburn \& Brewster [9] sont a priori les seuls à avoir étudié l'impact de distracteurs multiples sur un retour haptique et une technique agissant dans l'espace moteur (sticky targets) pour une tâche écologique (sélection dans un menu). Les résultats de toutes ces expériences suggèrent un impact négatif des distracteurs sur le temps de pointage, le taux d'erreur ou la satisfaction des utilisateurs, tous les auteurs recommandant une étude plus poussée de cet impact.

\section{STIMTAC ET LA TECHNIQUE SURFPAD}

La méconnaissance de l'impact des distracteurs sur les techniques de facilitation du pointage et les perspectives ouvertes par les nouvelles technologies haptiques sont à l'origine de notre travail. Notre technique Surfpad repose ainsi sur un dispositif particulier : le STIMTAC.

Le STIMTAC est un pavé tactile à retour tactile basé sur l'effet de film comprimé décrit précédemment. Il est constitué de 28 céramiques piézoélectriques collées sous une plaque de cuivre-béryllium de $82 \times 49 \mathrm{~mm}$ [5]. La plaque vibre sous l'effet de la contraction/rétraction des céramiques à la fréquence ultrasonique de $36 \mathrm{kHz}$, n'émettant ainsi aucun bruit audible. Cette fréquence se situant en dehors de la bande-passante des mécanorécepteurs de la peau, la vibration n'est pas non plus perçue directement par l'utilisateur. La plaque semble par contre plus glissante à mesure que l'on augmente son amplitude. Le dispositif est typiquement configuré pour une amplitude maximale de $1 \mu \mathrm{m}$, la réduction de friction pouvant ainsi atteindre $50 \%$ selon l'état de la surface (la nettoyer modifie aussi son coefficient de friction, par exemple).

Les capteurs de position habituellement utilisés dans les pavés tactiles sont incomptabiles avec le principe de fonctionnement du STIMTAC : un capteur résistif atténuerait les vibrations et un capteur capacitif serait perturbé par le champ électrique créé. Un capteur optique spécifique est donc utilisé pour localiser le doigt de l'utilisateur. Basé sur deux LEDs blanches, un jeu de mirroirs et une barrette CCD, ce capteur offre une résolution de 170 dpi. Un DSP calcule les barycentres de deux ombres créées sur la barrette CCD par le doigt posé sur la plaque et les transmet à $120 \mathrm{~Hz}$ sous forme de coordonnées $(x, y)$ sur une liaison série. Cette liaison permet de spécifier le coefficient de friction désiré en envoyant un réel compris entre 0.0 (aucun effet, friction maximale) et 1.0 (effet maximum, friction minimale) à une fréquence maximum de $400 \mathrm{~Hz}$.
Le retour tactile produit par le STIMTAC s'appuie sur la rugosité perçue par le mouvement du doigt sur la plaque. Un utilisateur déplaçant son doigt sur celle-ci inactive la sentira lisse, non collante. Mais en raison de son coefficient de friction élevé, la peau sera étirée de manière notamment évidente lors des changements de direction. L'activation de l'effet de film comprimé ne modifie en rien les caractéristiques intrinsèques de la plaque. Mais la réduction de friction qu'elle entraine diminue l'étirement de la peau, produisant un effet similaire au toucher d'un morceau de soie. Si l'effet de film comprimé est désactivé alors que le doigt se déplace sur la surface, l'augmentation de la friction est clairement remarquable.

Considérant ces caractéristiques, nous nous sommes intéressés à la manière dont le STIMTAC pouvait être utilisé pour faciliter le pointage. Comme nous l'avons vu, la plupart des techniques sensibles aux cibles modifient les caractéristiques du mouvement dans le monde virtuel (pour les techniques dans l'espace moteur) ou dans le monde réel (pour les techniques haptiques) : elles ralentissent généralement le pointeur ou guident son déplacement. D'une certaine manière, on peut considérer que ces techniques augmentent la friction liée au pointage à proximité des cibles. Le STIMTAC, lui, ne peut que réduire la friction. Pour l'augmenter en certains endroits, il faut donc la diminuer partout ailleurs. La technique que nous proposons résulte ainsi d'une inversion figure/fond : au lieu de ralentir le pointeur à proximité des cibles, pourquoi ne pas faciliter son déplacement là où il n'y en a pas ? Notre technique Surfpad utilise ainsi l'effet de film comprimé de STIMTAC pour réduire le coefficient de friction de la plaque lorsque le pointeur est en dehors des cibles et le laisser à son niveau normal dans le cas contraire.

\section{EXPÉRIENCE 1}

Le but de cette première expérience était d'évaluer l'effet de notre technique Surfpad sur une tâche de pointage simple sans distracteur en la comparant à une adaptation de gain sensible aux cibles et à un gain constant. La technique d'adaptation de gain retenue est celle du pointage sémantique [6].

\section{Matériel, tâche et sujets}

L'expérience s'est déroulée sur un PC équipé d'un moniteur LCD 15 " $1280 \times 800$, d'un clavier et du STIMTAC utilisé comme périphérique d'entrée pour les trois techniques. Écrite en $\mathrm{C}++$, l'application assurait un rendu graphique et haptique respectivement à 60 et $400 \mathrm{~Hz}$.

Le protocole utilisé est celui du pointage unidimensionnel réciproque. La cible à sélectionner (une bande verticale) était affichée en vert et devenait grise lorsqu'on la sélectionnait, la cible suivante apparaissant alors de l'autre côté de l'écran. Un son était joué lorsqu'un sujet manquait la cible, et une erreur enregistrée. Le sujet devait nécessairement sélectionner la cible désignée pour pouvoir passer à la suivante, même s'il devait pour cela s'y reprendre à plusieurs fois. Le pointeur, une ligne verticale de 1 pixel 
de large, n'était pas confiné à la partie visible de l'écran afin de ne pas permettre une utilisation particulière de ses bords (aucune sortie de l'écran n'a été observée durant l'expérience). Les sujets utilisaient la touche Ctrl du clavier avec leur deuxième main pour sélectionner les cibles.

Douze bénévoles agés de 26 ans en moyenne ont pris part à cette expérience ( 11 hommes et 1 femme, 10 droitiers et 2 gauchers).

\section{Conditions et procédure}

Un plan intra-sujets et une analyse à mesures répétées ont été utilisés. Les variables indépendantes étaient la technique utilisée (Technique), la distance à la cible (Distance) et sa taille (TAILle). Les distances possibles étaient $\mathrm{D}_{\mathrm{G}}=100 \mathrm{~mm}, \mathrm{D}_{\mathrm{M}}=50 \mathrm{~mm}$ et $\mathrm{D}_{\mathrm{P}}=25 \mathrm{~mm}$. Les tailles possibles étaient $\mathrm{T}_{\mathrm{G}}=4,136 \mathrm{~mm}=16$ pixels, $\mathrm{T}_{\mathrm{M}}=2,068 \mathrm{~mm}=8$ pixels et $\mathrm{T}_{\mathrm{P}}=1,034 \mathrm{~mm}=4$ pixels. L'indice de difficulté de la tâche de pointage se situait ainsi dans l'intervalle $[2,8-6,6]$. Les techniques possibles étaient un gain constant sans activation du STIMTAC (Contrôle), le Pointage Sémantique et Surfpad.

Dans leur expérience sur le pointage sémantique, Blanch et al. avaient utilisé un gain de base de 1 [6]. Nous avons pour notre part choisi un gain de 2 afin que les trois techniques puissent être utilisées sans débrayage quelle que soit la distance, compte tenu des dimensions du STIM$\mathrm{TAC}^{1}$. Pour le pointage sémantique, nous quadruplons la taille des cibles dans l'espace moteur en utilisant la fonction en cloche $\Omega$ décrite dans [6], cette configuration donnant les meilleurs résultats selon les auteurs.

Les sujets disposaient de quelques minutes dans la condition Contrôle pour s'habituer au STIMTAC avant de débuter l'expérience. Ils devaient ensuite exécuter 4 BLOCS de 27 essais pour chaque technique : 3 répétitions des 9 combinaisons possibles DistanCE $\times$ TAILLE, les valeurs de ces deux variables étant présentées par ordre décroissant. L'ordre de présentation des techniques était contrebalancé entre les sujets en utilisant un carré latin. Les sujets étaient encouragés à faire une pause tous les 9 essais, l'expérience durant environ 25 minutes.

Le plan de l'expérience était donc le suivant : 12 sujets $\times$ 3 TEChNiques $\times 4$ Blocs $\times(3$ Distances $\times 3$ TAILles $)$ $\times 3=3888$ essais au total.

\section{RÉSULTATS DE L'EXPÉRIENCE 1}

Les principales variables dépendantes sont le taux d'erreur et le temps de pointage, que nous décomposons ensuite afin mieux comprendre les effets observés.

\section{Taux d'Erreur}

Le taux d'erreur observé est de 4,1\%. Une ANOVA à mesures répétées montre un effet significatif de TAILLE $\left(\mathrm{F}_{2,22}=30,5, \mathrm{p}<0,001\right)$ sur ce taux, celui-ci augmentant à mesure qu'elle diminue ( $\left.\mathrm{T}_{\mathrm{P}}: 8,3 \%, \mathrm{~T}_{\mathrm{M}}: 2,7 \%, \mathrm{~T}_{\mathrm{G}}: 1,4 \%\right)$.

1. Débrayer consiste à désactiver temporairement le contrôle du pointeur par une action sur le périphérique, en le soulevant par exemple.

\section{Temps de Pointage}

Le temps de pointage est défini comme le temps mis pour aller d'une cible à la suivante et la sélectionner. Les cibles pour lesquelles une erreur avait été enregistrée ont été ignorées pour son analyse. Les essais à plus de trois déviations standards de la moyenne pour chaque condition TECHNIQUE $\times$ DistancE $\times$ TAILlE ont également été ignorés (1,6\% des essais).

Une ANOVA à mesures répétées montre l'absence d'effet et d'interaction liés à l'ordre de présentation des techniques. On observe par contre un effet significatif de BLOCS $\left(\mathrm{F}_{3,33}=7,8, \mathrm{p}=0,001\right)$. Une comparaison deux à deux ${ }^{2}$ montre des différences significatives $(\mathrm{p}<0,02)$ entre les deux premiers blocs et les deux derniers. Les deux premiers ont donc été ignorés dans le reste de l'analyse.

Une ANOVA à mesures répétées montre un effet significatif de Technique $\left(\mathrm{F}_{2,22}=10,1, \mathrm{p}=0,001\right)$. Des comparaisons deux à deux montre des différences significatives entre Surfpad et Contrôle $(1,51 \mathrm{~s}$ vs. $1,79 \mathrm{~s}, \mathrm{p}=0,012)$ et entre Pointage Sémantique et Contrôle $(1,45 \mathrm{~s}$ vs. $1,79 \mathrm{~s}, \mathrm{p}=0,001)$, la différence entre Pointage Sémantique et Surfpad n'étant pas significative $(\mathrm{p}=0,43)$. Surfpad améliore les performances de $15,5 \%$ comparé à Contrôle, tandis que Pointage Sémantique les améliore de 18,9\%.

Comme prédit par la loi de Fitts, on observe des effets principaux significatifs de DISTANCE $\left(\mathrm{F}_{2,22}=55,8, \mathrm{p}<0,001\right)$ et TAILLE $\left(\mathrm{F}_{2,22}=90,0, \mathrm{p}<0,001\right)$, et une interaction significative DistanCE $\times$ TAILLE $\left(\mathrm{F}_{4,44}=9,4, \mathrm{p}<0,001\right)$. On observe surtout une interaction TECHNIQUE $\times$ TAILLE $\left(\mathrm{F}_{4,44}=18,2\right.$, $\mathrm{p}<0,001)$. Des comparaisons deux à deux montrent des différences significatives entre les techniques à mesure que la taille diminue. Pour $\mathrm{T}_{\mathrm{G}}$, il n'y a pas de différence significative entre les techniques ( $p>0,59)$. Pour $T_{M}$, des différences significatives existent entre Surfpad et Contrôle $(1,40$ s vs. $1,80 \mathrm{~s}, \mathrm{p}=0,04)$ et entre Pointage Sémantique et Contrôle $(1,39 \mathrm{~s}$ vs. $1,80 \mathrm{~s}, \mathrm{p}<0,001)$. Pour $\mathrm{T}_{\mathrm{P}}$, des différences significatives existent également entre Surfpad et Contrôle $(1,75 \mathrm{~s}$ vs. $2,18 \mathrm{~s}, \mathrm{p}=0,02)$ et entre Pointage Sémantique et Contrôle (1,58s vs. $2,18 \mathrm{~s}, \mathrm{p}=0,001)$.

Afin de mieux comprendre les effets observés sur le temps de pointage, nous le décomposons dans ce qui suit en trois temps (approche, arrêt et sélection) et nous intéressons également au temps passé en mode débrayé.

\section{Temps d'Approche}

Le temps d'approche est celui mis pour atteindre le bord de la cible. On observe sur ce temps un effet principal significatif de TeChNiQue $\left(\mathrm{F}_{2,22}=12,5, \mathrm{p}<0,001\right)$, Distance $\left(\mathrm{F}_{2,22}=157,7, \mathrm{p}<0,001\right)$ et TAILlE $\left(\mathrm{F}_{2,22}=33,2, \mathrm{p}=0,001\right)$, et des interactions significatives TECHNIQUE $\times$ TAILlE $\left(\mathrm{F}_{4,44}=58,7, \mathrm{p}<0,001\right)$ et Distance $\times$ TAILlE $\quad\left(\mathrm{F}_{4,44}=29,1\right.$, $\mathrm{p}<0,001)$. Des comparaisons deux à deux montrent des différences significatives entre Contrôle et Surfpad ou Pointage Sémantique $(0,97 \mathrm{~s}$ vs. 0,85 s pour les deux autres,

2. Toutes les comparaisons post-hoc ont été faites en utilisant la méthode d'ajustement de Bonferroni. 
$\mathrm{p}<0,001)$. Des comparaisons deux à deux montrent également que le temps d'approche augmente à mesure que la distance augmente ou que la taille diminue.

L'interaction TECHNIQUE $\times$ TAILLE montre un comportement particulier du Pointage Sémantique sur $\mathrm{T}_{\mathrm{G}}$, avec un temps d'approche plus important que Contrôle et Surfpad $(0,97 \mathrm{~s}$ vs. $0,84 \mathrm{~s}$ et $0,74 \mathrm{~s}, \mathrm{p}<0,001)$. Ceci explique peut-être pourquoi aucune différence significative n'a été trouvée entre le pointage sémantique et Contrôle pour le temps de pointage.

\section{Temps d'Arrêt}

Le temps d'arrêt est celui entre le moment où le pointeur traverse pour la première fois le bord de la cible et celui où il s'arrête. Une ANOVA à mesures répétées montre un effet principal significatif de BLOCS sur ce temps $\left(\mathrm{F}_{3,33}=4,8\right.$, $\mathrm{p}=0,008$ ). Des comparaisons deux à deux montrent des différences significatives $(\mathrm{p}<0,03)$ entre les deux premiers blocs et les deux derniers, ce qui pourrait expliquer l'effet d'apprentissage observé sur le temps de pointage. Les deux premiers blocs ont donc été ignorés pour la suite de l'analyse.

On observe également un effet principal significatif de TeChnique $\left(\mathrm{F}_{2,22}=3,8, \mathrm{p}<0,038\right)$. Des comparaisons deux à deux montrent une différence significative entre Contrôle et Surfpad (0,56s vs. 0,43s, p=0,02, Pointage Sémantique étant à $0,47 \mathrm{~s}$ ). On observe aussi un effet principal significatif de TAILlE $\left(\mathrm{F}_{2,22}=17,5, \mathrm{p}=0,001\right)$, le temps d'arrêt augmentant à mesure qu'elle diminue. Des comparaisons deux à deux montrent une différence significative entre les trois tailles $\left(\mathrm{T}_{\mathrm{P}}=0,59 \mathrm{~s}, \mathrm{~T}_{\mathrm{M}}=0,49 \mathrm{~s}, \mathrm{~T}_{\mathrm{G}}=0,38 \mathrm{~s} ; \mathrm{p}<0,013\right)$.

\section{Temps de Sélection}

Le temps de sélection est le temps pendant lequel le pointeur reste immobile une fois arrêté avant que la touche Ctrl ne soit enfoncée. On observe des effets significatifs de Technique $\left(\mathrm{F}_{2,22}=23,9, \mathrm{p}<0,001\right)$ et TAILle $\left(\mathrm{F}_{2,22}=80,6\right.$, $\mathrm{p}=0,001)$ sur ce temps ainsi qu'une interaction significative Technique $\times$ Taille $\left(\mathrm{F}_{4,44}=4,8, \mathrm{p}<0,003\right)$. Des comparaisons deux à deux montrent des différences significatives $(\mathrm{p}<0,001)$ entre Pointage Sémantique $(0,185 \mathrm{~s})$, Contrôle $(0,258 \mathrm{~s})$ et Surfpad $(0,260 \mathrm{~s})$. On observe également des différences significatives entre les tailles $\left(T_{P}\right.$ $\left.=0,30 \mathrm{~s}, \mathrm{~T}_{\mathrm{M}}=0,23 \mathrm{~s}, \mathrm{~T}_{\mathrm{G}}=0,17 \mathrm{~s} ; \mathrm{p}<0,001\right)$. L'interaction montre des différences significatives $(\mathrm{p}<0,005)$ entre Pointage Sémantique et les deux autres techniques, quelle que soit la taille.

\section{Temps de Débrayage}

Le temps de débrayage durant un essai est celui pendant lequel le doigt ne touchait pas le STIMTAC. On observe un effet significatif de Distance $\left(\mathrm{F}_{2,22}=18,6, \mathrm{p}<0,001\right)$ et TAILlE $\left(\mathrm{F}_{2,22}=8,6, \mathrm{p}=0,002\right)$ sur ce temps, des comparaisons deux à deux montrant qu'il augmente avec ces deux variables. Des comparaisons deux à deux montrent une différence significative entre $\mathrm{T}_{\mathrm{G}}$ et $\mathrm{T}_{\mathrm{P}}(0,093 \mathrm{~s}$ vs. $0,073 \mathrm{~s}$, $\mathrm{p}=0,013)$ et entre $\mathrm{T}_{\mathrm{G}}$ et $\mathrm{T}_{\mathrm{M}}(0,093 \mathrm{~s}$ vs. $0,070 \mathrm{~s}, \mathrm{p}=0,015)$.
On observe un effet principal significatif de TeCHNIQUE $\left(\mathrm{F}_{2,22}=4,8, \mathrm{p}=0,02\right)$, Distance $\left(\mathrm{F}_{2,22}=15,3, \mathrm{p}<0,001\right)$ et TAILLE $\left(\mathrm{F}_{2,22}=9,7, \mathrm{p}=0,001\right)$ et une interaction significative Technique $\times$ Distance $\left(\mathrm{F}_{4,44}=3,4, \mathrm{p}<0,016\right)$ sur le nombre de débrayages $\mathrm{N}_{\text {Deb }}$. Des différences significatives existent entre Pointage Sémantique et Contrôle et Surfpad (0,73 vs. 0,35 et $0,37, \mathrm{p}<0,05)$. Comme prévisible, $\mathrm{N}_{\text {Deb }}$ augmente avec la distance avec une différence significative entre $D_{P}, D_{M}$ et $D_{G}(0,22,0,43$ et 0,$81 ; p<0,05)$. Il augmente aussi avec la taille des cibles, avec une différence significative entre $T_{P}$ et $T_{G}(0,44$ vs. $0,56, p=0,007)$.

\section{Analyse en termes de loi de Fitts}

Nous avons analysé le temps de pointage en termes de loi de Fitts en ne conservant que les deux derniers blocs et en ignorant les essais durant lesquels une erreur ou un débrayage s'était produit. Pour le pointage sémantique, nous avons calculé l'indice de difficulté dans l'espace moteur, comme suggéré dans [6]. Nous avons agrégé les données pour chaque distance et taille, produisant ainsi un total de 9 points pour chaque technique. Nous obtenons ainsi de bons coefficients de corrélation pour Contrôle $\left(M T=0,22+0,30 I D, r^{2}=0,92\right)$ et Surfpad $\left(M T=0,35+0,23 I D, r^{2}=0,95\right)$ et, dans une moindre mesure, Pointage Sémantique $(M T=0,78+0,19 I D$, $\left.r^{2}=0,78\right)$. Ce dernier résultat s'explique principalement par un point aberrant $\left(\mathrm{D}_{\mathrm{G}}, \mathrm{T}_{\mathrm{G}}\right)$ par ailleurs source du plus grand nombre de débrayages. Bien que les essais concernés ne soient pas pris en compte ici, nous supposons que ces débrayages ont perturbé la rythmicité des mouvements des sujets.

\section{Retour Utilisateur}

La plupart des sujets ont indiqué avoir ressenti avec Surfpad un retour tactile sur les cibles de taille $\mathrm{T}_{\mathrm{P}}$ et $\mathrm{T}_{\mathrm{M}}$ et un effet de freinage sur $\mathrm{T}_{\mathrm{G}}$. Ils pensaient avoir été plus précis dans ce dernier cas. Les sujets ont tous indiqué avoir eu l'impression que le pointeur était attiré par les cibles avec le pointage sémantique et qu'ils étaient plus performants avec cette technique qu'avec les deux autres. En réponse à la question "quelle technique choisiriez-vous pour une utilisation quotidienne ?", six ont répondu le pointage sémantique, quatre Surfpad et deux Contrôle.

\section{EXPÉRIENCE 2}

Les résultats de l'expérience 1 montrent que Surfpad et le pointage sémantique réduisent de manière significative le temps de pointage, particulièrement dans le cas des petites cibles. Le but de cette seconde expérience était de voir si les deux techniques conservent leur avantage face à Contrôle en présence de distracteurs.

\section{Matériel, tâche et sujets}

Le matériel et le logiciel utilisés étaient le même que dans l'expérience 1. La tâche était également la même, à l'exception de distracteurs équitablement espacés sur le chemin de la cible à sélectionner. Douze bénévoles agés de 28 ans en moyenne ont pris part à cette expérience (10 hommes et 2 femmes, 9 droitiers et 3 gauchers). 


\section{Conditions et procédure}

Un plan intra-sujets et une analyse à mesures répétées ont été utilisés. Les variables indépendantes étaient la technique utilisée (TECHNIQUe), la taille des cibles (TAILLE) et la densité des distracteurs (DENSITÉ). Les techniques possibles étaient Contrôle, Pointage Sémantique et Surfpad. Les tailles possibles étaient $\mathrm{T}_{\mathrm{G}}=4,136 \mathrm{~mm}=16$ pixels et $\mathrm{T}_{\mathrm{P}}=1,034 \mathrm{~mm}=4$ pixels. Les densités possibles étaient $0,1,2,4,8$ et 12 , les distracteurs étant de taille $\mathrm{T}_{\mathrm{G}}$ dans toutes les conditions. La distance à la cible était maintenue constante à $100 \mathrm{~mm}$ au cours de l'expérience afin de pouvoir évaluer l'impact des différentes densités de distracteurs tout en limitant le débrayage.

Les sujets disposaient de quelques minutes dans la condition Contrôle pour s'habituer au STIMTAC avant de débuter l'expérience. Ils devaient ensuite exécuter 4 séries (BLOCS) de 36 essais pour chaque technique : 3 répétitions des 12 combinaisons possibles TAILlE $\times$ DENsité. Les différentes tailles étaient présentées par ordre décroissant et les densitées par ordre croissant. L'ordre de présentation des techniques était contrebalancé entre les sujets en utilisant un carré latin. Les sujets étaient encouragés à faire une pause tous les 6 essais, l'expérience durant environ 35 minutes.

Le plan de l'expérience était donc le suivant : 12 sujets $\times$ 3 TeChNiques $\times 4$ Blocs $\times(2$ TAILles $\times 6$ Densités $)$ $\times 3=5184$ essais au total.

\section{RÉSULTATS DE L’EXPÉRIENCE 2}

Les variables dépendantes sont le taux d'erreur, le temps de pointage et le temps de débrayage.

\section{Taux d'erreur}

Une ANOVA à mesures répétées montre un effet significatif de TAILlE $\left(\mathrm{F}_{1,11}=96,3, \mathrm{p}<0,001\right)$ sur ce taux avec des différences significatives entre $\mathrm{T}_{\mathrm{P}}(10,2 \%)$ et $\mathrm{T}_{\mathrm{G}}(1,9 \%)$ et un taux moyen de $6,1 \%$.

\section{Temps de Pointage}

Les cibles pour lesquelles une erreur avait été enregistrée ont été ignorées pour cette analyse. Considérant les essais à plus de trois déviations standards de la moyenne pour chaque condition comme marginaux $(1,5 \%$ des essais), nous les avons également ignorés.

Une ANOVA à mesures répétées montre l'absence d'effet et d'interaction liés à l'ordre de présentation des techniques. On ne trouve pas non plus d'effet ou d'interaction de BLocs, ce qui indique l'absence d'effet d'apprentissage. Comme prédit par la loi de Fitts, on observe des effets principaux significatifs de TAILLE $\left(\mathrm{F}_{1,11}=199,4\right.$, $\mathrm{p}<0,001)$ sur le temps avec une augmentation de celui-ci pour les plus petites cibles.

On observe des effets significatifs de TeChniQue $\left(\mathrm{F}_{2,22}\right.$ $=119,1, \mathrm{p}<0,001)$ et Densité $\left(\mathrm{F}_{5,55}=67,8, \mathrm{p}<0,001\right)$ ainsi qu'une interaction significative TECHNIQUE $\times$ DENSITÉ $\left(\mathrm{F}_{10,110}=92,6, \mathrm{p}<0,001\right)$. Des comparaisons deux à deux montrent des différences significatives $(p<0,001)$ entre toutes les techniques (2,1s pour Contrôle, 2,9s pour Pointage Sémantique et 1,9s pour Surfpad). Surfpad améliore donc les performances de $9,5 \%$ par rapport à Contrôle, et de $52,6 \%$ par rapport au pointage sémantique, tandis que celui-ci dégrade les performances de 38,1\% par rapport à Contrôle.

Des comparaisons deux à deux pour l'interaction TECHNIQUE $\times$ DENSITÉ montrent que la dégradation de performance pour le pointage sémantique augmente avec DENSITÉ. Aucune différence n'est observée entre les techniques pour la densité 0 , mais on observe des différences significatives $(\mathrm{p}<0,04)$ entre Contrôle et Surfpad pour les densités supérieures à 1 . Des différences significatives $(\mathrm{p}<0,03)$ sont observées pour tous les niveaux de Densité pour le pointage sémantique, sauf entre 0 et $1(\mathrm{p}=0,16)$ et entre 2 et $4(\mathrm{p}=0,07)$. Aucune différence n'est observée entre les différents niveaux de DENSITÉ pour Contrôle et Surfpad.

\section{Temps de Débrayage}

Une ANOVA à mesures répétées montre un effet significatif de TeChNiQue $\left(\mathrm{F}_{2,22}=64,4, \mathrm{p}<0,001\right)$ et DENSITÉ $\left(\mathrm{F}_{5,55}=164,4, \mathrm{p}<0,001\right)$ ainsi qu'une interaction significative TECHNIQUE $\times$ DENSITÉ $\left(\mathrm{F}_{10,110}=121,9, \mathrm{p}<0,001\right)$ sur le temps de débrayage. Des comparaisons deux à deux montrent des différences significatives $(\mathrm{p}<0,001)$ entre Pointage Sémantique $(0,59 \mathrm{~s})$, Surfpad $(0,12 \mathrm{~s})$ et Contrôle $(0,15 \mathrm{~s})$. Aucune différence n'est observée pour les différents niveaux de DENsité avec Surfpad ou Contrôle, mais des différences significatives $(\mathrm{p}<0,003)$ sont observées entre les différents groupes de densités pour le pointage sémantique : $(0,1): 0,28 \mathrm{~s},(2): 0,42 \mathrm{~s},(4): 0,54 \mathrm{~s}$, $(8,12): 1,02 \mathrm{~s}$.

\section{Retour Utilisateur}

Les commentaires des sujets sur Surfpad et Contrôle sont similaires à ceux de l'expérience 1. La plupart des commentaires sur le pointage sémantique concerne le débrayage nécessaire pour déplacer le pointeur, particulièrement lorsque le nombre de distracteurs est important. Les sujets n'ont fait aucun commentaire spontané sur les distracteurs et Surfpad. Une fois interrogés, ils ont expliqué ne pas avoir eu l'impression d'être dérangés dans leur mouvement par le retour tactile sur ceux-ci. En réponse à la question "quelle technique choisiriez-vous?", onze ont répondu Surfpad et un Contrôle.

\section{DISCUSSION}

L'expérience 1 visait à comparer Surfpad à Contrôle et au pointage sémantique en utilisant le même gain de base. Les résultats obtenus montrent que Surfpad améliore le temps de pointage de 15,5\% comparé à Contrôle. Cette amélioration est proche de la différence de 18,9\% observée pour le pointage sémantique. Les résultats montrent aussi que ces différences peuvent être expliquées par une diminution du temps d'approche à la fois pour Surfpad et le pointage sémantique comparé à Contrôle, une différence du temps d'arrêt pour Surfpad comparé à Contrôle, 
et une différence du temps de sélection pour le pointage sémantique comparé à Contrôle et Surfpad.

L'expérience 2 visait à comparer à nouveau Surfpad à Contrôle et au pointage sémantique, mais en ajoutant un contrôle sur la densité des distracteurs. Les résultats montrent que Surfpad améliore le temps de sélection de 9,5\% par rapport à Contrôle, ceci indépendamment de la densité des distracteurs. Les résultats montrent également que le pointage sémantique dégrade le temps de pointage par rapport à Contrôle de manière d'autant plus forte que la densité de distracteurs est importante. Les résultats montrent enfin que l'augmentation du débrayage pour le pointage sémantique comparé aux deux autres techniques peut être tenue pour responsable de cette dégradation.

\section{Importance de la Taille de la Cible}

Nous observons dans l'expérience 1 un effet significatif de la taille de la cible sur le temps de pointage. Comparé à Contrôle, le temps de pointage moyen pour les cibles de tailles $\mathrm{T}_{\mathrm{M}}$ et $\mathrm{T}_{\mathrm{P}}$ est réduit de $21 \%$ pour Surfpad et $25,1 \%$ pour le pointage sémantique. Pour ce dernier, la réduction est d'autant plus importante que la cible est petite : de $22,8 \%$ pour $\mathrm{T}_{\mathrm{M}}$ à $27,5 \%$ pour $\mathrm{T}_{\mathrm{P}}$. Cependant, les améliorations de performances observées pour Surfpad et le pointage sémantique sont réduites à néant pour $\mathrm{T}_{\mathrm{G}}$ alors même que selon Blanch et al., le pointage sémantique réduit l'indice de difficulté dans l'espace moteur indépendamment de la taille de la cible [6].

Un point à noter est l'augmentation significative du débrayage avec les trois techniques pour $\mathrm{T}_{\mathrm{G}}$. Dans leur expérience Blanch et al. utilisaient un puck sur une tablette graphique faisant approximativement la même taille que l'écran. Bien que ce ne soit pas précisé dans leur article, nous pouvons supposer qu'aucun débrayage n'a eu lieu dans leur expérience. Nous faisons également l'hypothèse que le nombre plus important de débrayages observé dans l'expérience 1 pour $\mathrm{T}_{\mathrm{G}}(6,6 \%$ du temps de pointage) rend la détection d'effets significatifs entre les techniques plus difficiles à observer. D'autres expériences seraient nécessaires pour valider ces hypothèses.

\section{Distracteurs et Débrayage}

L'expérience 2 montre que la présence de distracteurs peut dégrader les performances du pointage sémantique de manière importante. Pour cette technique, l'augmentation de la taille des distracteurs dans l'espace moteur augmente la distance physique à parcourir, et donc le débrayage. La dégradation de performance pour le pointage sémantique par rapport à Contrôle augmente de $22,4 \%$ pour une densité de 2 et jusqu'à 100\% pour une densité de 12 .

Dans le pire scénario imaginable pour le pointage sémantique, la distance qui sépare le pointeur de la cible est complètement couverte de distracteurs. Dans ce cas, si les distracteurs ne se recouvrent pas, les indices de difficulté dans les espaces moteur et visuel deviennent égaux mais la distance dans l'espace moteur est multipliée. Si l'espace de travail du périphérique d'entrée est déterminé de façon à pouvoir couvrir la totalité de l'écran sans débrayage, cette multiplication conduira inévitablement à du débrayage et à une dégradation des performances.

Nous n'avons pas noté d'effet négatif des distracteurs pour Surfpad. Quel que soit leur nombre, le temps de pointage avec cette technique reste significativement meilleur de 9,5\% comparé à Contrôle. Les sujets n'ont pas émis de commentaire négatif sur le retour tactile associé aux distracteurs. Surfpad semble donc être une bonne alternative au pointage sémantique et sans doute aux autres techniques d'adaptation du gain sensibles aux cibles, particulièrement lorsque l'espace de travail est limité.

\section{Perception de la Distorsion de l'Espace Moteur}

Alors que Blanch et al. avaient indiqué que leurs sujets ne savaient pas dire s'ils utilisaient le pointage sémantique ou non, les sujets de notre première expérience ont signalé un effet d'attirance vers les cibles avec cette technique, quelle que soit leur taille. Avec un gain par défaut de 1 (le choix de Blanch et al.) et des cibles quadruplées dans l'espace moteur, le gain chute à 0,25 ce qui représente un différentiel de 0,75 . Avec un gain de base de 2 (notre choix), il chute à 0,5 soit un différentiel de 1,5 . Pour expliquer les différences de retour utilisateur entre l'expérience de Blanch et al. et la nôtre, nous faisons l'hypothèse que les utilisateurs sont plus sensibles au différentiel qu'au rapport entre les gains. D'autres expériences seront nécessaires pour valider cette hypothèse.

\section{Effet Mécanique ou Retour d'Information?}

On observe dans l'expérience 1 une diminution significative du temps d'approche pour le pointage sémantique comparé à Contrôle. La fonction en cloche $\Omega$ opérant uniquement au voisinage immédiat des cibles et ne faisant rien pour réduire ce temps, nous supposons que cet effet est la conséquence d'un phénomène d'anticipation déjà observé dans l'utilisation des cibles grossissantes.

L'expérience 1 montre également une diminution significative du temps d'approche de Surfpad comparé à Contrôle, proche de celle observée pour le pointage sémantique. Deux hypothèses peuvent être envisagées pour expliquer cela : (H1) des déplacements rendus mécaniquement plus rapides par la réduction de friction; ou (H2) des mouvements plus rapides à nouveau causés par une anticipation. Il est à noter que ces raisons sont compatibles et toutes deux positives puisque l'anticipation implique la perception d'un effet bénéfique ultérieur lié à Surfpad.

L'expérience 1 montre une réduction significative du temps d'arrêt pour Surfpad comparé à Contrôle. Deux hypothèses peuvent à nouveau expliquer cela : (H3) un effet de freinage mécanique lié à l'augmentation de la friction; ou (H4) un retour d'information tactile, une hypothèse confortée par des travaux antérieurs qui montrent que l'ajout d'information tactile peut réduire les temps de réponse en fournissant une confirmation ne nécessitant pas d'attention visuelle $[2,13]$. 
Surfpad étant plus efficace sur les petites cibles où il devrait être plus difficile de profiter de l'effet de freinage, nous privilégions l'hypothèse $\mathrm{H} 4$ pour expliquer la réduction du temps d'arrêt. Cette préférence est confortée par les commentaires des utilisateurs concernant le retour tactile pour $\mathrm{T}_{\mathrm{P}}$ et $\mathrm{T}_{\mathrm{M}}$ et l'effet de freinage pour $\mathrm{T}_{\mathrm{G}}$. Elle est également appuyée par les résultats de l'expérience 2 durant laquelle les distracteurs n'ont pas dégradé les performances de Surfpad : un effet mécanique aurait ralenti les utilisateurs et donc augmenté le temps de pointage.

Notons que toutes nos hypothèses sont compatibles : Surfpad pourrait fournir une assistance mécanique utile dans les phases d'approche (H1) et d'arrêt (H3) ainsi qu'un retour d'information (H4) favorisant l'anticipation (H2).

\section{CONCLUSION}

Nous avons présenté Surfpad, une nouvelle technique de facilitation du pointage basée sur STIMTAC, un pavé tactile au coefficient de friction ajustable. Nous avons présenté les résultats de deux expériences comparant Surfpad au pointage sémantique et à un gain constant. Nos résultats montrent que le gain de temps permis par Surfpad face au pointage sans assistance peut aller jusqu'à $21 \%$ dans le cas de petites cibles sans distracteur. Notre technique est également robuste aux distracteurs, améliorant en moyenne les performances de près de $10 \%$ quand le pointage sémantique peut les dégrader jusqu'à $100 \%$. De nouvelles expériences sont actuellement en cours pour approfondir notre compréhension de la technique.

\section{REMERCIEMENTS}

Ce travail a été en partie financé par le projet ANR-09CORD-013 "InSTInCT".

\section{BIBLIOGRAPHIE}

1. Akamatsu, M., and MacKenzie, I. S. Movement characteristics using a mouse with tactile and force feedback. IJHCS, 45(4) :483-493, 1996.

2. Akamatsu, M., MacKenzie, I. S., and Hasbrouc, T. A comparison of tactile, auditory, and visual feedback in a pointing task using a mouse-type device. Ergonomics, 38(4) :816-827, 1995.

3. Balakrishnan, R. "Beating" Fitts' law : virtual enhancements for pointing facilitation. IJHCS, 61(6) :857-874, 2004.

4. Baudisch, P., Cutrell, E., Czerwinski, M., Robbins, D. C., Tandler, P., Bederson, B. B., and Zierlinger, A. Drag-and-pop and drag-and-pick : techniques for accessing remote screen content on touch- and penoperated systems. In Proc. of Interact'03, 57-64. IOS Press, 2003.

5. Biet, M., Giraud, F., and Semail, B. Squeeze film effect for the design of an ultrasonic tactile plate. IEEE Transactions on Ultrasonic, Ferroelectric and Frequency Control, 54(12) :2678-2688, 2007.

6. Blanch, R., Guiard, Y., and Beaudouin-Lafon, M. Semantic pointing : improving target acquisition with control-display ratio adaptation. In Proc. of CHI'04, 519-526. ACM, 2004.
7. Campbell, C. S., Zhai, S., May, K. W., and Maglio, P. P. What you feel must be what you see : adding tactile feedback to the trackpoint. In Proc. of Interact'99, 383-390. IOS Press, 1999.

8. Chapuis, O., Labrune, J., and Pietriga, E. Dynaspot : speed-dependent area cursor. In Proc. of CHI'09, 1391-1400. ACM, 2009.

9. Cockburn, A., and Brewster, S. Multimodal feedback for the acquisition of small targets. Ergonomics, 48(9) :1129-1150, 2005.

10. Cockburn, A., and Firth, A. Improving the acquisition of small targets. In Proc. of HCI'03, 77-80. BCS, 2003.

11. Dennerlein, J. T., Martin, D. B., and Hasser, C. Force-feedback improves performance for steering and combined steering-targeting tasks. In Proc. of CHI'00, 423-429. ACM, 2000.

12. Dennerlein, J. T., and Yang, M. C. Haptic forcefeedback devices for the office computer : performance and musculoskeletal loading issues. Human Factors, 43(2) :278-286, 2001.

13. Forlines, C., and Balakrishnan, R. Evaluating tactile feedback and direct vs. indirect stylus input in pointing and crossing selection tasks. In Proc. of CHI'08, 1563-1572. ACM, 2008.

14. Guiard, Y., Blanch, R., and Beaudouin-Lafon, M. Object pointing : a complement to bitmap pointing in GUIs. In Proc. of GI'04, 9-16. CHCCS.

15. Harrison, C., and Hudson, S. E. Texture displays : a passive approach to tactile presentation. In Proc. of CHI'09, 2261-2264. ACM, 2009.

16. Hwang, F., Keates, S., Langdon, P., and Clarkson, P. Multiple haptic targets for motion-impaired computer users. In Proc. of CHI'03, 41-48. ACM, 2003.

17. Keyson, D. V. Dynamic cursor gain and tactual feedback in the capture of cursor movements. Ergonomics, 40(12) :1287 - 1298, 1997.

18. Lécuyer, A., Burkhardt, J.-M., and Etienne, L. Feeling bumps and holes without a haptic interface : the perception of pseudo-haptic textures. In Proc. of CHI'04, 239-246. ACM, 2004.

19. Oakley, I., McGee, M. R., Brewster, S., and Gray, P. Putting the feel in 'look and feel'. In Proc. of CHI'O0, 415-422. ACM, 2000.

20. Watanabe, T., and Fukui, S. A method for controlling tactile sensation of surface roughness using ultrasonic vibration. In Proc. of ICRA'95, 1134-1139. IEEE.

21. Winfield, L., Glassmire, J., Colgate, J. E., and Peshkin, M. T-pad : Tactile pattern display through variable friction reduction. Proc. of World Haptics Conference, 421-426, 2007.

22. Worden, A., Walker, N., Bharat, K., and Hudson, S. Making computers easier for older adults to use : area cursors and sticky icons. In Proc. of CHI'97, 266-271. ACM. 